\title{
A printed millimeter-wave modulator and antenna array for low-complexity Gigabit-datarate backscatter communications
}

\author{
Spyridon Daskalakis ( $\sim$ Daskalakispiros@gmail.com ) \\ Heriot-Watt University \\ Apostolos Georgiadis \\ Heriot-Watt University https://orcid.org/0000-0003-0054-8167 \\ John Kimionis \\ Nokia Bell Labs \\ Manos Tentzeris \\ Georgia Institute of Technology
}

\section{Article}

Keywords: materials, devises, electrical devices

Posted Date: January 21st, 2021

DOI: https://doi.org/10.21203/rs.3.rs-144028/v1

License: (c) This work is licensed under a Creative Commons Attribution 4.0 International License. Read Full License

Version of Record: A version of this preprint was published at Nature Electronics on June 10th, 2021. See the published version at https://doi.org/10.1038/s41928-021-00588-8. 


\title{
A printed millimeter-wave modulator and antenna array for low-complexity Gigabit-datarate backscatter communications
}

\author{
John Kimionis ${ }^{1}$, Apostolos Georgiadis ${ }^{2}$, Spyridon Nektarios Daskalakis ${ }^{2, *}$, and Manos M. \\ Tentzeris $^{3}$
}

\author{
${ }^{1}$ Nokia Bell Labs, Murray Hill, NJ 07974, USA \\ ${ }^{2}$ Herriot-Watt University, School of Engineering and Physical Sciences, Edinburgh, EH14 4AS, UK \\ ${ }^{3}$ Georgia Institute of Technology, School of Electrical and Computer Engineering, Atlanta, GA 30308, USA \\ *Corresponding author. email: daskalakispiros@gmail.com
}

\begin{abstract}
In this article, a low cost ink-jet printed millimeter-wave RF front-end for low-complexity Gigabit-datarate backscatter communications was designed, fabricated and measured. The RF front-end consists of a microstrip $5 \times 1$ series-fed patch antenna array and a single E-pHEMT transistor, supporting a plethora of modulation formats, including binary phase shift keying (BPSK), quadrature phase shift keying (QPSK), and quadrature amplitude modulation (16-QAM). The circuit was additively manufactured using inkjet printing with silver nanoparticle (SNP) inks on a flexible liquid crystal polymer (LCP) substrate. A mmWave transceiver was also designed in order to capture and downconvert the backscattered signals and route them for digital signal processing. A bit rate of $2 \mathrm{Gbps}$ of backscatter transmission is demonstrated at millimeter-wave frequencies $24-28$ $\mathrm{GHz}$, expanding the potential of backscatter radio as an ambitious low-energy, low-complexity communication system for future IoT devices. By pushing the circuit complexity to a central station/access point, the radio's footprint is minimized, which allows additive manufacturing, resulting in significant implementation savings and compatibility with flexible platforms. The wideband operation of these systems will enable broadband wireless transmission with less than $0.17 \mathrm{pJ} /$ bit front-end consumption at $2 \mathrm{Gbps}$ and combined with sensing with low-power sensors and can be integrated with wearables for challenging mobile applications in $5 \mathrm{G}$ and the Internet of Things (IoT).
\end{abstract}

\section{Introduction}

As Internet of Things (IoT) tends to become commonplace in everyone's daily life, there is a necessity for more scalable and robust communication systems. IoT sensor devices are increasing/evolving every day and all that concerns the communications go in the same direction, which translates to a requirement for higher data rates and lower power consumption per device. Backscatter radios will play a crucial role in the future due to their low cost, low complexity, and battery-free operation ${ }^{1-4}$. Therefore, there is a significant need to design novel wireless communication techniques to achieve higher data rates ${ }^{5-7}$ while simultaneously minimizing energy consumption.

Millimeter wave (mmWave) communications have traditionally been considered as the "the last mile" providing broadband, directive point-to-point and point-to-multipoint wireless links with applications such as Local Multipoint Distribution Systems (LMDS). Notable advantages of this technology are the wide available bandwidth in the order of GHz, enabling very large communication rates, as well as the ability to implement electrically large antenna arrays albeit being physically compact, due to the small wavelength that allows beamforming capabilities. However, to date such mmWave systems feature significantly high cost in terms of components as well as system fabrication cost due to typical requirements for low-loss materials and small-footprint assembly processes. At the same time, mmWave systems dissipate more power than their corresponding counterparts operating in the ultra high frequency (UHF) or low-microwave frequency range (e.g. 1-5 GHz electronics that are commonly available in commercial devices). This is due primarily to limitations in device technologies leading to imperfect switching operation (diodes, transistors), but also due to layout parasitic resulting from fabrication and packaging tolerances effectively deteriorating the obtained efficiency and finally due to losses in dielectric and conducting materials in the operating frequencies. However, lower-frequency electronics are limited in terms of datarate due to the drastically smaller fractional bandwidth they can support.

Over the last five years, there has been an increasing need for higher datarate transmission from fixed stations, mobile devices, and Internet-of-Things (IoT) nodes, which has paved the way to fifth generation (5G) communications and direct adoption of mmWave technologies to support wideband operation. In this article, we are exploring the option of utilizing 
reflection-communication, namely backscatter radio for Gigabit-datarate mmWave communications, while minimizing the front-end complexity to a single high frequency transistor. This gives direct benefits in fabrication time and cost, as well as tremendous energy footprint reduction per communication node. Backscatter radio has been extensively used in commercial radio frequency identification (RFID) applications in sub-GHz bands to wirelessly transmit static identification codes with kbps-level datarates. Millimeter wave identification (MMID) systems were initially proposed in ${ }^{8}$ leveraging the advantages of directional antennas with beam scanning capability used in the reader, permitting localization and ranging functionality. In addition, the possibility for short range, high bit rate communication due to a larger available bandwidth was recognized, but was not demonstrated. Another advantage of millimeter wave operation that has been explored in the past is the direct integration of the antennas on integrated circuits ${ }^{9}$, especially for the implementation of miniaturized implantable applications. Implementations of various reader architectures and tag systems have been published exploring different technologies, from pure $\mathrm{CMOS}^{9-14}$ to a combination of CMOS and $\mathrm{LTCC}^{15}$, to $\mathrm{BiCMOS}^{16}$, to silicon micromachining ${ }^{9}$ and to tag circuits consisting of off-the-shelf Schottky diodes placed on liquid crystal polymer substrate ${ }^{17}$.

Prior art has demonstrated backscatter rates of $100 \mathrm{~s}$ of Mbps in the $2.4 \mathrm{GHz}$ frequency range as well as up to $24 \mathrm{GHz}$. Even though the advantages of using mmWave bands for low-complexity data transmission have been acknowledged by the prior art, no ultra-high-datarate backscatter operation has been reported until ${ }^{15,18,19}$. Moreover, prior art has been limited to binary modulation only ${ }^{15}$. Here, backscatter radio is shown to be a key enabler for lower-cost mmWave communications, while successful conveying of dynamic data in multi-Gbit backscatter transmissions is demonstrated for the first time improving the state-of-the-art data rates by 2-3 orders of magnitude in a low power of $0.17 \mathrm{pJ} / \mathrm{bit}$. More importantly, it is shown for a first time that a single mmWave transistor can support a plethora of modulation formats, including binary phase shift keying (BPSK), quadrature phase shift keying (QPSK), and quadrature amplitude modulation (16-QAM).

Analog modulation of the backscatter signal has been proposed in the literature, for example $\mathrm{in}^{20,21}$. In our work we explore analog modulation by introducing an analog control signal in the gate of a commercial off-the-shelf transistor. We have demonstrated a $4 \mathrm{Gbps}$ binary modulation transmission for the first time, to the best of our knowledge, in ${ }^{15}$ and in this work we demonstrate transmission of a Gbps higher order complex modulation generated with the same single transistor hardware as ${ }^{15}$ and present the received signal constellation in real time using commercial test equipment. The work in ${ }^{15}$ presented preliminary results of a front-end with less than $0.15 \mathrm{pJ} / \mathrm{bit}$ energy consumption at $4 \mathrm{Gbps}$ and combining communication and sensing capability. While the preliminary results demonstrated the capability to transmit a Gbps modulated waveform at millimeter waves, this work further shows that the transmitted waveform presents a signal to noise and distortion level which is sufficient to implement complex modulation formats with high spectral efficiency, by demonstrating a received Gbps 16-QAM signal constellation. In fact the demonstrated modulation rate performance has been limited by the available bandwidth of the receiver test equipment hardware. In this work we present different backscatter signal constellations (BPSK, QPSK, and 16-QAM) and additionally we provide a brief theoretical analysis of backscattering and power consumption.

The low complexity of the front-end developed for the Gbit-datarate backscatter demonstration is very appealing as it allows for the first time an additive manufacturing using inkjet printing techniques. Ink-jet printing, which has been used for rapidly fabricating mmWave antennas ${ }^{22}$ and packaging solutions ${ }^{23}$, inherently requires minimal amounts of material and achieves micron-level fabrication accuracy. In this work, additive manufacturing has enabled fast, yet high-performance, prototyping which drastically reduces the fabrication cost and lead time typically associated with fab houses commonly utilized for mmWave communication systems.

\section{Single-transistor high-order modulation}

Figure 1 summarizes the concept of single-transistor Gbit backscatter communications at mmWave frequencies. A backscatter communicator consists of an antenna array with input impedance $Z_{\mathrm{a}}$ and a single $\mathrm{RF}$ transistor that is used for modulation with impedance $Z$. By controlling the RF transistor's gate bias with a voltage $V_{\mathrm{b}}(t)$, the transistor impedance can be easily modified, yielding a variation of the reflection coefficient over time. The complex reflection coefficient between the antenna array input impedance and the voltage-controlled transistor impedance is

$$
\Gamma\left(V_{b}(t)\right)=\frac{Z\left(V_{b}(t)\right)-Z_{a}^{*}}{Z\left(V_{b}(t)\right)+Z_{a}}
$$

and denotes the relationship between the RF voltage $V_{\mathrm{RF}}^{\text {refl }}$ that is reflected, when an incident RF voltage $V_{\mathrm{RF}}^{\text {inc }}$ is induced at the antenna's terminal, as it is evident from $V_{\mathrm{RF}}^{\text {refl }}=\Gamma V_{\mathrm{RF}}^{\mathrm{inc}}$. The complex function $\Gamma$ can carry digital information (e.g. a bitstream), in a similar way that a complex baseband communication signal digitally encodes information in a conventional transmitter. For example, binary data in typical RFIDs is encoded by transitioning between two $\Gamma_{0}$ and $\Gamma_{1}$ values, when voltages $V_{0}$ and $V_{1}$ respectively, are applied to the transistor gate.

This article introduces an exceptional difference in comparison to prior art backscatter modulators, that enables high-order constellation (non-binary) possible, with the use of a single transistor. Conceptually, multiple transistors or cascaded RF 


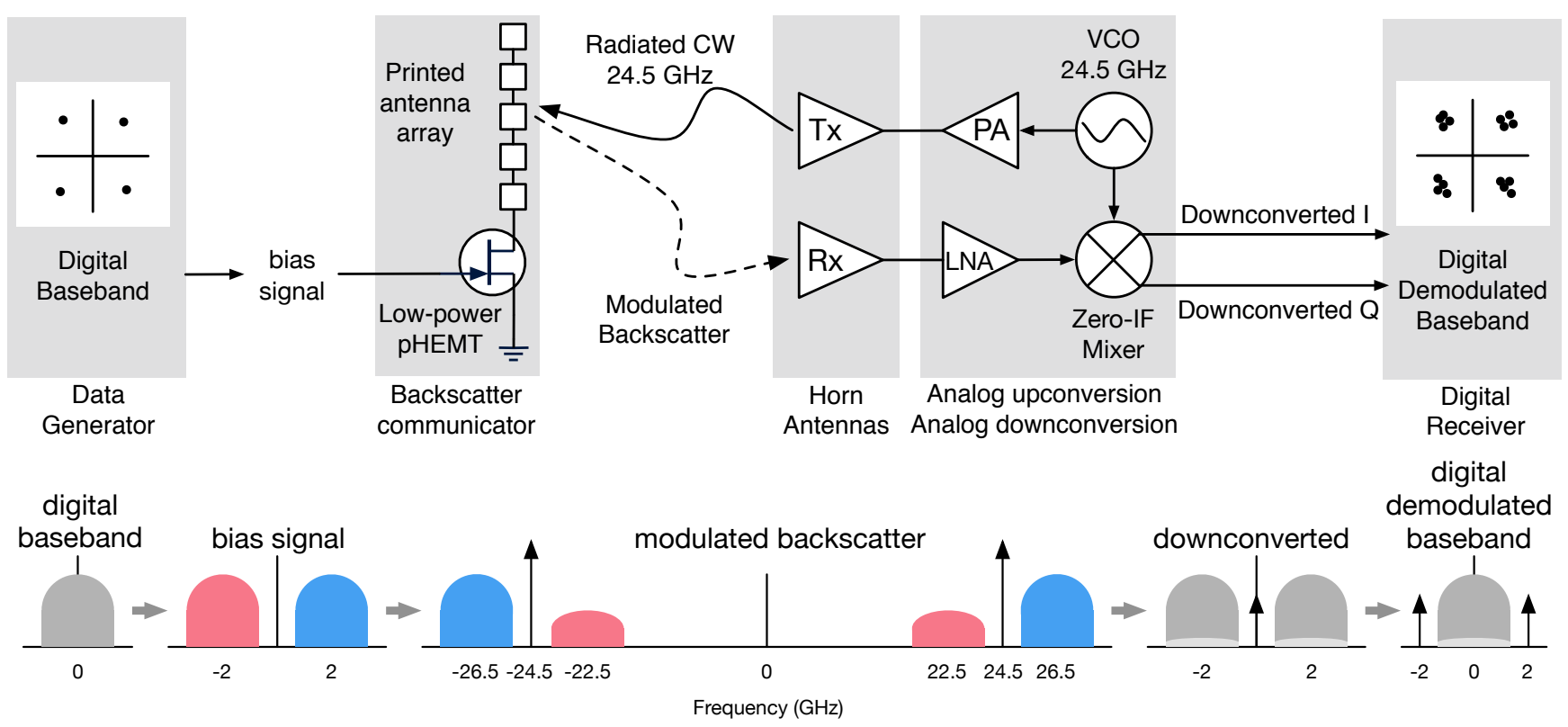

Figure 1. Top: Gbps-datarate mmWave backscatter system with $24-28 \mathrm{GHz}$ transceiver and single-transistor backscatter communicator. The single-transistor communicator acts as a wireless mixer that is illuminated with an unmodulated continuous wave $(\mathrm{CW})$ signal by the transceiver and mixes high-speed data by controlling the transistor's impedance. The transceiver receives the modulated backscattered signals and demodulates them for digital base-band processing. Bottom: Heterodyne modulation and demodulation of Gbit-datarate mmWave backscatter. Grey lobes correspond to spectra of complex-valued signals.

multiplexers could be used in different topologies to generate multiple reflection coefficient values, as has been showcased for UHF QAM backscatter in ${ }^{1}$ and ${ }^{16,24}$. However, increasing the number of transistors in a mmWave implementation may:

- decrease performance due to multiple device parasitics ${ }^{16}$

- increase cost

- increase complexity for higher constellation sizes, as seen in ${ }^{1}$, which limits scalability, whereas

- using switching-mode devices results in unwanted, increased spectrum occupancy, as argued $\mathrm{in}^{25}$.

An important result of this work is that it applies the principles of continuously modulating the backscattered signal as it was shown in analog backscattering publications such $a^{20}$ and our previous work related to pulse shaping of the backscattered signal $^{25}$.

Modern communication devices push the signal processing complexity toward the digital baseband IC, instead of e.g. employing multiple mixer stages in the RF front-end hardware. Radios tend to be more software-defined, leaving room for communication protocol upgrades and modulation scheme reconfiguration, while reutilizing unchanged, generic RF front-ends and antennas. The same philosophy is followed here, by keeping one single antenna array and one modulation transistor operating in mmWave bands, while pushing the complexity to lower-frequency digital operations.

The complex baseband signal $x(t)$ in this implementation is a wideband (500 MHz-1 GHz main lobe width) BPSK, QPSK, or 16-QAM modulation, which is digitally upconverted to a $f_{\text {sub }}=2 \mathrm{GHz}$ subcarrier, yielding a real-valued IF signal

$$
x_{b}(t)=\Re\left\{x(t) e^{j 2 \pi f_{\text {sub }} t}\right\}
$$

which is used to bias the transistor's gate. Since the baseband signal is digitally mixed with a subcarrier, and the IF signal is real-valued, only one transistor will always need to be excited, regardless of the baseband signal's number of dimensions, which guarantees a feature that enables easy scalability.

When the backscatter communicator is illuminated with a $24.5 \mathrm{GHz}$ unmodulated continuous wave (CW), it reflects a wave that relies on the amplitude and phase of $\Gamma$, effectively mixing the IF signal around the $24.5 \mathrm{GHz}$ carrier. This wireless mixing process enables mmWave transmissions completely eliminating the need for mmWave oscillators and mixers on the 

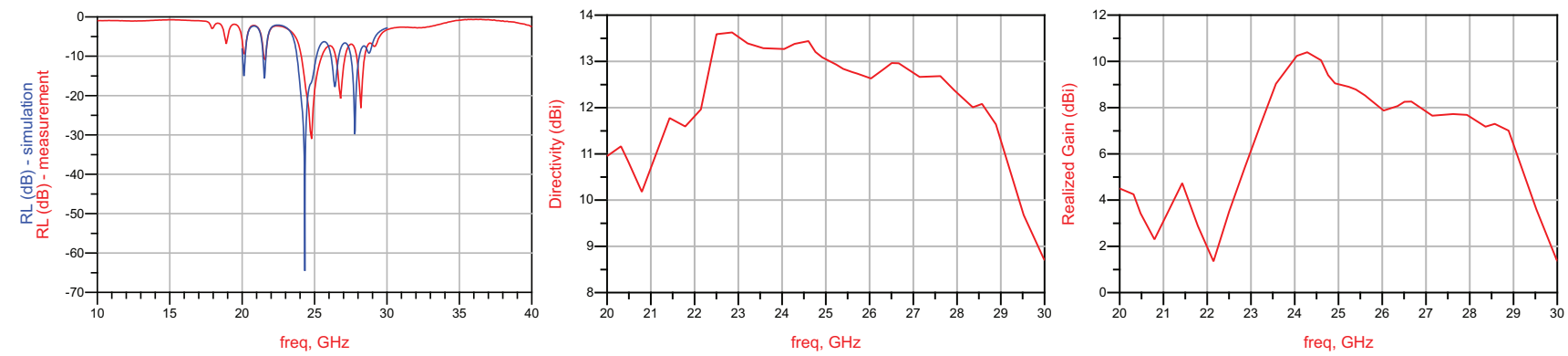

Figure 2. Left: Simulated and measured array return loss (RL). Center: Simulated $5 \times 1$ array directivity. Right: Simulated $5 \times 1$ array realized gain.

communicator. The result is a passband mmWave signal with upper and lower sidebands that is captured by a $24.5-\mathrm{GHz}$ receiver and directly downconverted to dc with a mixer that is coherent with the $24.5 \mathrm{GHz}$ transmitter that emits the carrier $\mathrm{CW}$. The in-phase (I) and quadrature (Q) components of the mixer output are routed to a digital receiver that is digitally removing the $2 \mathrm{GHz}$ IF and demodulating the received complex baseband constellation. Employing a zero-IF downcoverter in the mmWave hardware offers the flexibility of selecting any bias signal IF, without the need for additional mixing stages either in the backscatter communicator, or the mmWave transceiver. The advantage of this heterodyne backscatter is that within the bandwidth capability of the hardware, any baud rate, IF, constellation size, and modulation format can be supported.
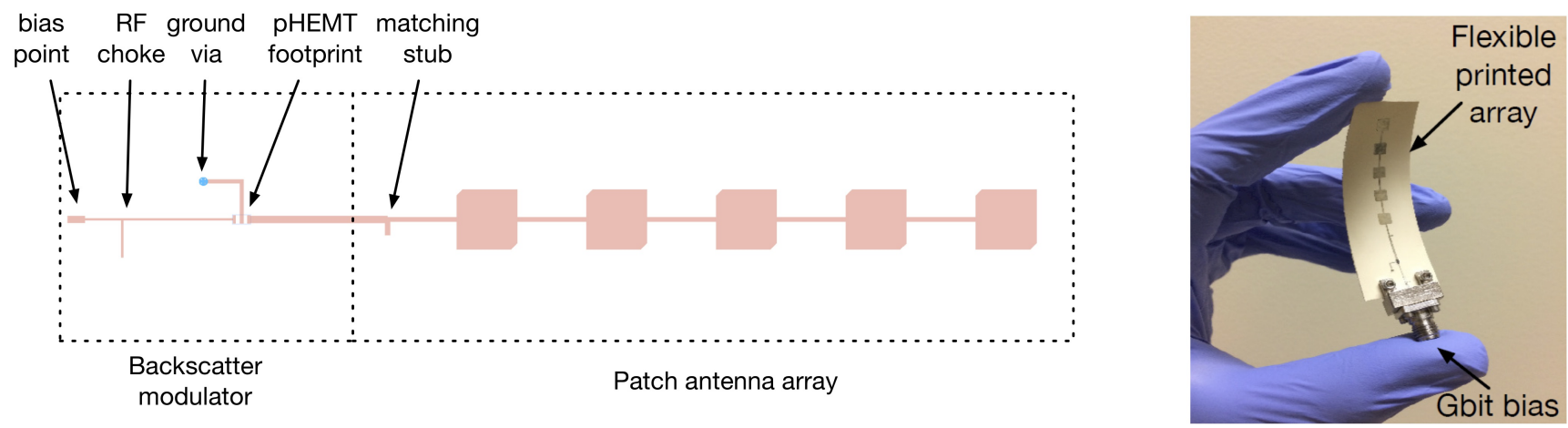

Figure 3. Left: Physical layout of the backscatter modulator and $5 \times 1$ antenna array physical layout. Right: mmWave backscatter communicator flexible prototype with an integrated pHEMT transistor front-end and patch antenna array.

\section{Printed mmWave backscatter communication array}

In principle, any antenna array can be used for mmWave backscattering, as long as it has a single feed that can be attached to a transistor's terminal. For demonstration purposes, a microstrip $5 \times 1$ series-fed patch antenna array is designed with 5 patch elements separated by transmission line sections of approximate length $\lambda_{\text {guided }} / 2$, where $\lambda_{\text {guided }}$ is the effective wavelength in the microstrip, leading to in-phase excitation of the patches and a resonance frequency of $24.5 \mathrm{GHz}$. The antenna array is circularly polarized and circular polarization is achieved by appropriately truncating two corners of the patch radiators ${ }^{26}$ The simulated and VNA-measured return loss of the array is shown in Figure 2-left, where the principal resonance can be seen around $24.5 \mathrm{GHz}$, and a secondary resonance can be seen around $26.5 \mathrm{GHz}$. The multiple-resonance frequency response is due to the fact that the series-fed antenna array has a structure of a bandpass microstrip stepped-impedance filter ${ }^{27,28}$. Due to the Bode-Fano limits, a broadband-response antenna array will feature multiple local minima in the return loss (RL) response.

The directivity (Figure 2-center) of the array remains approximately constant over the whole $23-28 \mathrm{GHz}$ band, with a value of approximately $13 \mathrm{dBi}$. The realized gain of the array (which includes the effect of the return loss, as well as conductor losses) can be seen in Figure 2-right; the gain at the center frequency $(24.5 \mathrm{GHz})$ is $10 \mathrm{dBi}$, and the gain between $25.5 \mathrm{and} 28 \mathrm{GHz}$ is flat at $8 \mathrm{dBi}$. The realized gain defines the frequency regions for the mmWave backscatter operation: the carrier $\mathrm{CW}$ that illuminates the communicator will be centered at $24.5 \mathrm{GHz}$, while the backscatter response (subcarrier) will be centered $2 \mathrm{GHz}$ further, at $26.5 \mathrm{GHz}$. An extra observation has to be made for the $22.5 \mathrm{GHz}$ region, where the realized gain is as low as $2 \mathrm{dBi}$. 
This frequency region inherently acts as a filter, which reduces the power of the lower modulated sideband that would otherwise act as a self-interfering image at the receiver. This "filtering effect" is illustrated in Figure 1-bottom, where the $22.5 \mathrm{GHz}$ lobe is shown as attenuated.

The antenna array layout along with the backscatter modulator front-end is shown in Figure 3. The antenna feed is connected through a $50 \Omega$ microstrip line to an E-pHEMT transistor's (Avago VMMK-1225 ${ }^{29}$ ) drain. The transistor's source has to be connected to an RF-short to achieve ideal reflection of $\Gamma=-1$ when biased and $\Gamma=+1$ when non-biased. At the same time, a dc-short is required to achieve bias signal return through the gate-source path and effectively create a $V_{\mathrm{GS}}>$ 0 bias voltage. Although a straightforward choice would be to place a grounded via as close as possible to the source pad, the physical dimension of the via would exceed the pad size effectively shorting the gate and the drain. This layout issue is alleviated by connecting the transistor's source to a grounded via through two approximately $\lambda / 4$ lines in series and in a gamma-configuration, that guarantee an unchanged impedance (RF short). The exact lengths of the lines have been optimized to achieve a $\lambda / 2$ total electrical length. The transistor's gate is connected to a bias feed (RF choke) which has been implemented with $\lambda / 4$ transformers, instead of parasitics-heavy lumped inductors. The utilized distributed RF choke features a rejection better than $25 \mathrm{~dB}$ at $24.5 \mathrm{GHz}$, which is the front-end's principal frequency of operation. That guarantees minimal carrier signal leakage to the gate and in turn to the bias bias Gbps source.

The integrated backscatter communicator has been implemented on 7-mil flexible liquid crystal polymer (LCP) substrate with one copper-clad side used as ground plane, while the other side (exposed LCP) is used for inkjet-printing the antenna array with silver nanoparticle (SNP) ink. The printing process is described in detail in the Methods section, and includes preparation of the LCP substrate, inkjet printing of the array and modulator circuit traces, followed by the attachment of the surface mount 0402 E-pHEMT. Due to the good adhesion of SNP on the thin LCP substrate, the complete structure is flexible, which can make it appealing for integration with wearable devices (e.g. smart wristbands, robots, autonomous cars and UAV's, space platforms and smart skins.). For a printed SNP conductivity value of $\sigma=5 \times 10^{6} \mathrm{~S} / \mathrm{m}$, the skin depth at $24 \mathrm{GHz}$ is $1.45 \mu \mathrm{m}$, which can be guaranteed by printing 4 layers of silver ${ }^{22}$. The microphotographs in Figure 4 show the high level of accuracy that is achieved with inkjet printing and four-layer stacking of SNP. The accuracy of the fine feature sizes as well as metal-to-metal clearance gaps are achieved by modifying the surface energy of LCP with UV ozone exposure, as described in the Methods section.
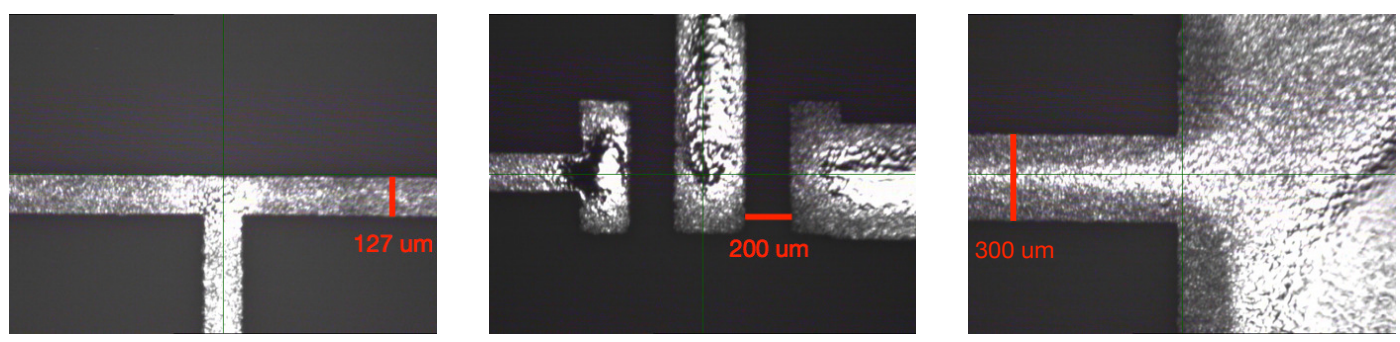

Figure 4. Microphotographs of inkjet-printed 24.5-GHz patch antenna array and backscatter communication circuit traces. Left: Shunt stub T-junction. Center: 0402 SMD pads for E-pHEMT transistor. Right: RF microstrip-to-patch transition.

\section{Custom mmWave backscatter transceiver}

The test setup of Figure 5 has been used to conduct the mmWave backscatter experiments. As shown in Figure 1-top, a mmWave transceiver is used to capture and downconvert the backscattered signals and route them for digital processing. For this work's testing, a custom transceiver with off-the-shelf modules has been set up, with a transmitter set up to emit a $24.5 \mathrm{GHz} \mathrm{CW}$. The spectrum of the receiver covers the whole $24-28 \mathrm{GHz}$ band, while it discards the $22.5 \mathrm{GHz}$ frequency region, further attenuating the lower modulated sideband, illustrated in Figure 1-bottom.

A voltage-controlled oscillator (VCO) (Hittite $\mathrm{HMC} 39^{30}$ ) is set up as a $24.5 \mathrm{GHz}$ synthesizer and its output is amplified by a power amplifier (PA) (Hittite HMC863 ${ }^{31}$ ) before it is sent to a wideband horn antenna (A-info LB-180400-20-C-KF) for CW illumination of the backscatter array. The horn gain was $20 \mathrm{~dB}$ and the PA provides $24 \mathrm{~dB}$ of gain, $+28.5 \mathrm{dBm}$ of saturated output power or $27 \mathrm{dBm}$ output power for $1 \mathrm{~dB}$ Compression at $+5.5 \mathrm{~V}$ supply. An identical horn antenna is used for the receive chain, connected to a mmWave low-noise amplifier (LNA) (RF-Lambda RLNA26G40GB ${ }^{32}$ ). The output of the LNA is connected to a zero-IF mixer (Hittite HMC977 ${ }^{33}$ ) with I/Q outputs.

In order to demonstrate the proof-of the proposed disruptive ultrabroadband backscatter communications, the mmWave communicator has been connected to a Keysight M8190 arbitrary waveform generator (AWG) that provides the real-valued modulated bias signal. The transceiver's I and Q outputs have been directed to a Keysight Infiniium S-series Digital Storage 


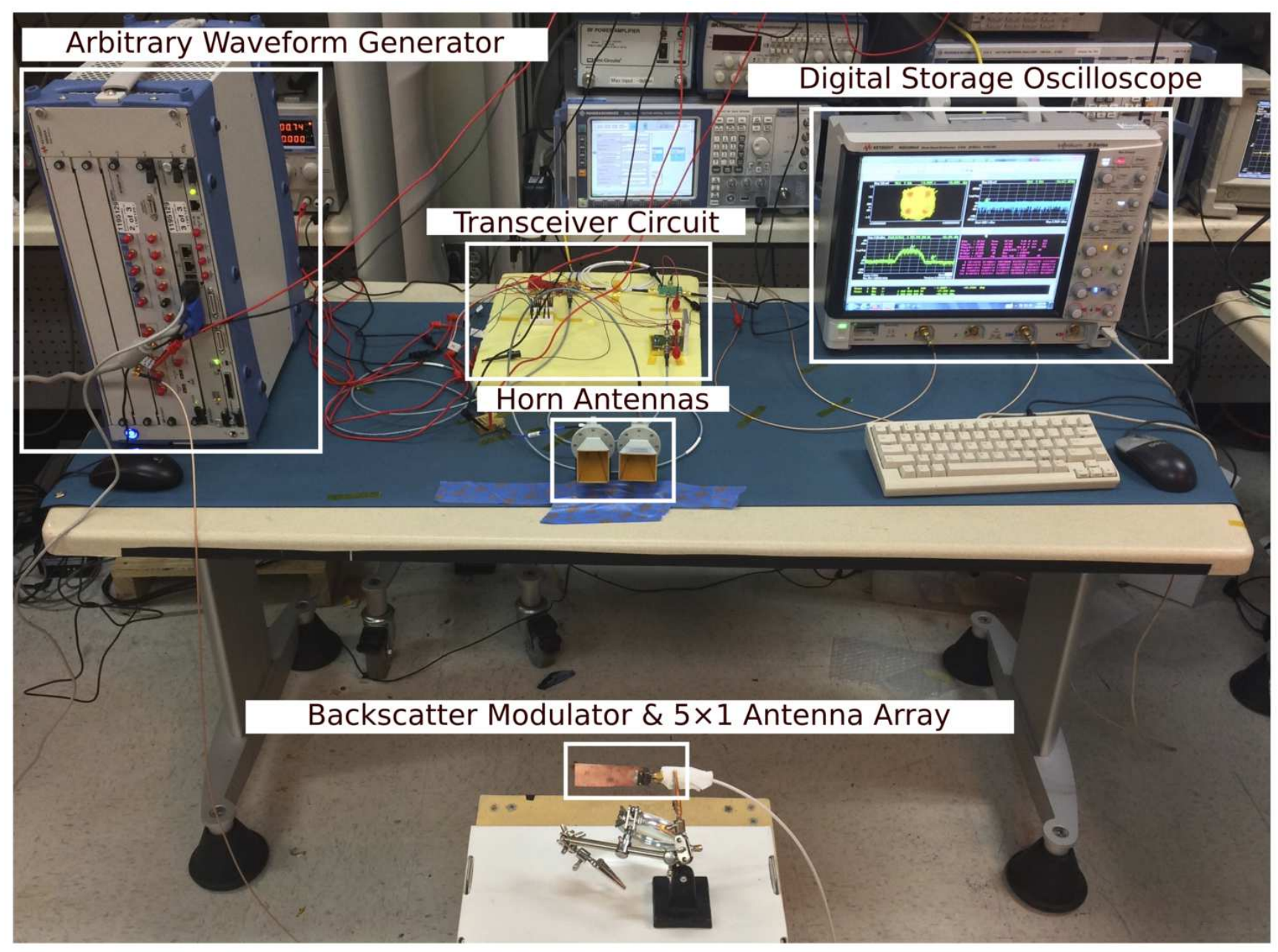

Figure 5. $\mathrm{Gb} / \mathrm{sec}$ mmWave backscatter setup comprising a waveform generator that drives the backscatter communicator, and a custom transmit and receive chain for illuminating the backscatter communicator, and decoding the reflected modulated signals.

Oscilloscope (DSO) running vector signal analysis (VSA) digital demodulation software and performing constellation analysis (Figure 5). The VSA receiver algorithm ${ }^{34}$ performs carrier and symbol synchronization and dc offset removal and rotation in order to generate a reference constellation. However, IQ imbalance is not compensated.

The distance between horn antennas and the backscatter RF front end was fixed at $0.5 \mathrm{~m}$. The linearly polarized transmit horn and received horn antennas were placed next to each other and rotated 90 degrees with respect to each other in order to receive linearly polarized signals that are orthogonally polarized with respect to the transmitted linearly polarized CW signal. This cross-polarized arrangement improves the isolation between the transmiter TX and the receiver RX and permits the system to reject structural scattering from the backscatter tag's ground plane ${ }^{22}$.

\section{Results}

The AWG is first set up to output BPSK pseudorandom data with a data rate of $1 \mathrm{Gbps}$ and a subcarrier of $2 \mathrm{GHz}$; that is the main lobe of the modulation bias covering the band from approximately $1.5 \mathrm{GHz}$ to $2.5 \mathrm{GHz}$. Instead of performing binary level switching between $0 \mathrm{~V}$ and $1 \mathrm{~V}$ which results in excessive-bandwidth signals, the pulse shaping principles of ${ }^{25}$ have been applied, to backscatter smooth, band-limited waveforms with most of the energy present in the main lobe. The front-end is biased with a BPSK waveform that is pulse-shaped with square-root raised cosine (SRRC) pulses with a rolloff factor $\alpha=0.35$ to limit the signal bandwidth. The demodulated signal can be seen in Figure 6, where the captured BPSK constellation and main lobe spectrum can be seen, with an RMS Error Vector Magnitude (EVM) of 24.76\%. This value corresponds to a bit error rate (BER) value of $0.2 \%{ }^{35}$ and has been achieved without any signal pre-distortion or forward error coding (FEC) at the 

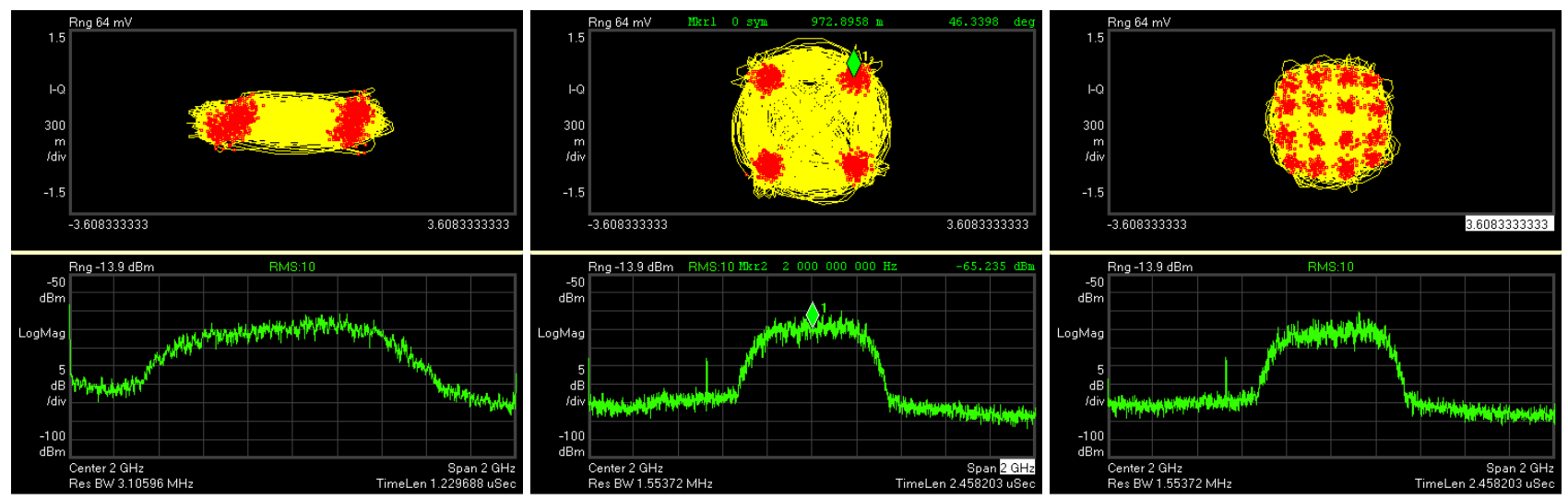

Figure 6. Demodulated Gigabit backscatter constellations and spectra. BPSK, QPSK, and 16-QAM.

AWG, and without equalization at the DSO/VSA. Considering the leakage gate-source current $I_{G S}=200 \mathrm{nA}$ and maximum bias voltage of $V_{\max }=1 \mathrm{~V}$, the maximum transistor static power consumption

$$
P_{\text {static }} \triangleq I_{\mathrm{GS}} * V_{\text {max }}
$$

is $P_{\text {static }}=200 \mathrm{nW}$. The maximum dynamic power consumption is ${ }^{36}$ :

$$
P_{d y n} \triangleq \frac{1}{2} C_{G S} V_{\text {max }}^{2} f_{\text {max }},
$$

where the gate capacitance is $C_{G S}=0.3 \mathrm{pF}$ and the maximum switching speed $f_{\max }=F_{\text {sub }}+W$ is related to the subcarrier $f_{\text {sub }}=2 \mathrm{GHz}$ and the baseband bandwidth $\mathrm{W}$ of the SRRC pulse used ${ }^{37}$ :

$$
W \triangleq \frac{1+\alpha}{2 T_{s y m}},
$$

where $\alpha=0.35$ is the SRRC rolloff factor and $T_{\text {sym }}$ is the symbol period. In the case of 1-Gbps BPSK, $f_{\max }=2.675 \mathrm{GHz}$, and $P_{d y n}=401.25 \mu \mathrm{W}$. The energy per bit

$$
E_{b} \triangleq\left(P_{\text {static }}+P_{\text {dyn }}\right) T_{b i t},
$$

where $T_{b i t}$ is the bit period, is then $E_{b}=0.4 \mathrm{pJ} / \mathrm{bit}$.

\begin{tabular}{c|c|c|c|c|c}
38 & 1 & 25 & 18 & 19 & THIS WORK \\
\hline \hline $900-930 \mathrm{MHz}$ & $900-930 \mathrm{MHz}$ & $900-930 \mathrm{MHz}$ & $2.45 \mathrm{GHz}$ & $2.45 \mathrm{GHz}$ & $\mathbf{2 4 - 2 8 ~ G H z}$ \\
\hline $4-\mathrm{QAM}$ & $16-\mathrm{QAM}$ & $4-\mathrm{PAM}$ & $16-\mathrm{QAM}$ & $16-\mathrm{QAM}$ & $\mathbf{1 6 - Q A M}$ \\
\hline $400 \mathrm{kbps}$ & $96 \mathrm{Mbps}$ & $100 \mathrm{kbps}$ & $120 \mathrm{Mbps}$ & $960 \mathrm{Mbps}$ & $\mathbf{2 ~ G b p s}$ \\
\hline $12.75 \mathrm{pJ} / \mathrm{bit}$ & $15.5 \mathrm{pJ} / \mathrm{bit}$ & $2.5 \mathrm{pJ} / \mathrm{bit}$ & $6.7 \mathrm{pJ} / \mathrm{bit}$ & $0.061 \mathrm{pJ} / \mathrm{bit}$ & $\mathbf{0 . 1 7} \mathbf{~ p J} / \mathrm{bit}$ \\
\hline \hline
\end{tabular}

Table 1. Comparison to prior art of high-order constellation backscatter.

To demonstrate the backscattering of high-order modulation with a single transistor, the AWG has been set up to generate a QPSK waveform, digitally upconverted to a $2 \mathrm{GHz}$ IF subcarrier, and the real-valued analog signal output has been used to drive the mmWave communicator. In Figure 6-center, the demodulated QPSK constellation is shown, with an EVM of 14.75\%, or BER of $0.6 \%$. The baudrate is 500 MSymbols/sec, with an effective bitrate of 1 Gbps. The energy-per-bit in this case is $E_{b}=0.35 \mathrm{pJ} /$ bit. In Figure 6-right, the bit rate is increased even more, with a baudrate of $500 \mathrm{MSymbols} / \mathrm{sec}$, but with a 16-QAM constellation, boosting the bitrate to $2 \mathrm{Gbps}$, an EVM of $12.37 \%$, a BER value of $8 \%$, and maximum energy-per-bit of $E_{b}=0.17 \mathrm{pJ} / \mathrm{bit}$. The $0.17 \mathrm{pJ} / \mathrm{bit}$ refers to the front-end only. An that an overall consumption evaluation of the tag should include the power dissipated in generating the Gbps modulation signal, which however presents a challenge in all such Gbps systems due to the high consumption of AWG (DACS and FPGA logic). This work clearly demonstrates the first-ever reported 
Gbps-level data rates for backscatter radio, accompanied by extremely low-energy front-end consumptions, as shown in Table 1. The fact that this performance can be achieved with all-printed antenna arrays of a small size $(5 \times 1)$ shows the great potential of using mmWave backscatter for low-complexity Gbit communication, without directly utilizing high-complexity mm-wave modules (VCOs, mixers, amplifiers) on mobile devices, sensors, or IoT nodes.

\section{Discussion}

Although millimeter wave propagation corresponds to an increased free space path loss compared to propagation using signals at lower frequencies in the UHF or low GHz frequency bands due to a smaller wavelength, the overall system path loss is offset by the significantly larger antenna gains. Furthermore, recent works ${ }^{39}$ have supported the fact that rain attenuation and atmospheric absorption at millimeter wave frequencies would not present a significant degradation of performance, especially when selecting specific low absorption frequency ranges such as the one around $28 \mathrm{GHz}$ for transmission. The range of backscatter communication systems such as RFID systems assuming free space propagation is estimated as ${ }^{40}$

$$
R \propto \lambda \sqrt{G_{t} G_{r}}
$$

where $G_{t}$ is the transmit reader/interrogator antenna gain, $G_{r}$ is the receive tag antenna gain and $\lambda$ the free space operating wavelength. The proportionality factor depends on the transmitted power, the sensitivity of the reader interrogator or the receive tag sensitivity, the polarization mismatch between the transmit and receive antennas and the impedance mismatch between the tag antenna and the tag transistor circuit. Therefore, assuming a fixed proportionality constant, scaling down the operating wavelength can be offset by scaling up by the square of the amount of the product of the transmit and receive antenna gains. For example, increasing the operating frequency by an order of magnitude, i.e. $10 \mathrm{~dB}$ (e.g. from $2.4 \mathrm{GHz}$ to $24 \mathrm{GHz}$ ) would require an increase of the combined transmit and receive antenna gains by two orders of magnitude, i.e. 20 dB. The system prototype that we have implemented comprises a transmit antenna with $20 \mathrm{~dB}$ of gain and a receive antenna with $10 \mathrm{~dB}$ of gain. A typical UHF reader antenna has $7 \mathrm{~dB}$ of gain and a typical UHF RFID tag $0 \mathrm{~dB}$ of gain. Compared to a typical UHF RFID scenario our prototype introduces an increase in the combined transmit and receive gain of 200 or $23 \mathrm{~dB}$. The frequency scaling from $915 \mathrm{MHz}$ to $24.5 \mathrm{GHz}$ is 26.8 or $14.3 \mathrm{~dB}$. This translates to approximately $3 \mathrm{~dB}$ reduction in the achievable range. The range reduction can be minimized by employing a larger tag antenna array. However, a more accurate comparison should consider the sensitivity of the tag, as well as the operating bit rate. The use of an active tag or exploring forms of energy harvesting by integrating for example solar cells together with the tag antenna ${ }^{41}$ may further assist the implementation of the system.

This work demonstrated for the first time the mechanisms of achieving multi-Gbps backscatter with miniaturized miniaturized minimal-energy minimal-complexity RF front-ends and antennas, combined with inkjet printing additive manufacturing on a flexible substrate in mmWave bands. The potential of $\mathrm{pJ} / \mathrm{bit}$ communication at those frequencies is promising, due to the fact that commonly utilized active RF modules for mmWave communication consume several orders of magnitude more power. Indicatively, the off-the shelf mmWave mixer HMC977 features a power consumption of 600-700 $\mathrm{mW}$, whereas the power consumption of the single-transistor backscatter front-end is on the order of $0.5 \mathrm{~mW}$, which corresponds to a $1000 \mathrm{X}$ improvement on power consumption. Viewing backscatter communication as "remote" mixing will lead to a drastically reduced complexity of commercial devices that require high-datarate communication.

With conventional technologies, cellphones will have to upgrade to full $28 \mathrm{GHz}$ radios in order to benefit from the upcoming $5 \mathrm{G}$ standards and reach Gigabit data transfer speeds. However, the $2.4 \mathrm{GHz}$ electronics that are already present in most current cellphones could act as an IF bridge between baseband and mmWave frequencies with the addition of a single mmWave transistor for over-the-air mixing. Backscattering at mmWave frequencies holds an excellent potential in bringing $5 \mathrm{G}$ network compatibility to devices without actually incorporating additional highly-complex and costly $28 \mathrm{GHz} \mathrm{Tx} / \mathrm{Rx}$ chains. Scenarios such as cellphone data offloading (e.g. sending photos/videos to cloud storage) at "hotspots" in cafes or other public places are in line with the concept of "wireless charging hotspots" that make their appearance in similar locations.

Utilizing single-transistor front-ends for backscatter mixing instead of active mmWave mixers has a direct effect on the communication radio's cost, which drops by one to two orders of magnitude (tens of dollars for an active mixer down to less than a dollar for a single transistor). These cost savings combined with the significantly smaller footprint of a minimal backscatter front-end will positively affect the total production cost of mobile devices, making wireless technology capable of rapid data transfer more accessible.

Moreover, by further optimization of reader sensitivity and the backscatter communicator's antenna gain, even higher order constellations can be supported, which in turn reduces the required symbol rate (and thus bandwidth) for a given bitrate. This will relax the bandwidth requirements of antennas and devices, and will reduce the device's power consumption even more due to lower switching speeds. Finally. the implementation of a wearable systems and small mobile devices provides additional challenges due to multipath and antenna bending and antenna misalignments that require further evaluation efforts, that may represent object of future work. 


\section{Appendix: Methods}

\section{Inkjet-printing fabrication of backscatter communicator}

The substrate used for the printed array fabrication is double-side copper-clad, 7-mil-thick Ultralam 3850HT liquid crystal polymer (LCP) from Rogers Corporation $\left(\varepsilon_{r}=3.14, \tan \delta=0.002\right)$, with one side of the copper cladding chemically etched off. Prior to printing, the LCP-exposed side of the substrate is cleaned by adhering and removing Kapton tape, which abrasively removes dust. The substrate is then exposed in a UV ozone cleaner for 10 seconds, to break down surface contaminants, such as grease, and modify the substrate's surface energy for improved ink adhesion.

All printing is performed with a Dimatix DMP-2831 material deposition inkjet printer using Suntronic EMD5730 inkjet ink, with a $40 \%$ nanosilver content in water-based solvent. Four layers of silver nanoparticle (SNP) ink are printed, at a $20 \mu \mathrm{m}$ drop-to-drop spacing and a 600 second inter-layer delay. The printer's print table is heated to a maximum of $60^{\circ} \mathrm{C}$, to promote solvent evaporation between layers. The resulting printed traces feature sharp edges, as shown in Figure 4.

The LCP substrate with the printed array is annealed in a mechanical convection oven at $180^{\circ} \mathrm{C}$ for 60 minutes, in order to evaporate remaining solvent and sinter the silver nanoparticles to form continuous conductive traces.

Vias are formed manually with a Dremel rotary tool driving a $0.5 \mathrm{~mm}$ drill bit. Metallization of vias is performed by deposition of MG Chemicals 8330 S Conductive epoxy $(0.0007 \Omega \cdot \mathrm{cm}$ resistivity). The same epoxy is used for adhesion of the surface mounted pHEMT transistor Avago VMMK-1225 on the printed silver traces.

For the bias signal excitation port, a screw-on end-launch SMA connector is used (Southwest 292-06A-6) which provides a coaxial-to-microstrip transition.

\section{Simulations}

For the simulations, the Keysight Advanced Design System (ADS) solver was used in order to optimise the backscatter modulator and $5 \times 1$ antenna array, and export the Figure 2-middle-right results. A full electromagnetic analysis with the method of moments was applied to backscatter modulator and $5 \times 1$ antenna array, in order to estimate the losses from the LCP substrate and SNP, the fringing fields and the electromagnetic coupling between ports. Next, harmonic-balance and large-signal analysis was employed, taking into account the non-linear behavior of the E-pHEMT transistor.

In the simulations, the PHEMT was biased through a microstrip-stub RF choke (rejection better than $25 \mathrm{~dB}$ at $24 \mathrm{GHz}$ ) and for a bias voltage of $0 \mathrm{~V}$ and $1 \mathrm{~V}$ respectively, the reflection coefficient values $\Gamma_{0}$ and $\Gamma_{1}$ were exported up to $40 \mathrm{GHz}$. Then, the reflection coefficient difference amplitude $\Delta \Gamma=\left|\Gamma_{0}-\Gamma_{0}\right|$ is calculated, which is known as the modulation factor that needs to be maximized to optimize backscatter communication performance ${ }^{42}$.

\section{Appendix: Derivations}

\section{Heterodyne mmWave backscatter signal model}

Detailed analysis and derivation of backscatter signal model can be found $\mathrm{in}^{43}$, where binary modulation is performed with a single transistor that alternates between two discrete states ('on'- 'off' switching when the transistor's gate bias is high or low). Moreover, analysis of backscatter modulation with pulse-shaped signals instead of hard level switching can be found in ${ }^{25}$, where a single transistor is used to generate arbitrary, smooth waveforms, by continuous variation of the transistor's gate bias over time. The following analysis shows how a single transistor can still be used to to backscatter signals corresponding to complex constellations, e.g. quadrature phase shift keying (QPSK) or 16-ary quadrature amplitude modulation (16-QAM).

A complex baseband QAM signal has the form:

$$
x(t)=I(t)+j Q(t) \triangleq \sum_{n} a_{n, I} g_{T}(t-n T)+j \sum_{n} a_{n, Q} g_{T}(t-n T),
$$

where $I(t)$ and $Q(t)$ correspond to the in-phase and quadrature signal components, respectively, $g_{T}(t)$ is the transmit filter pulse (e.g. a square root raised cosine - SRRC with a baseband bandwidth $W$ ), $T$ is the symbol period, and $a_{n, I}, a_{n, Q}$ are the $n$-th in-phase and quadrature symbol to be transmitted. For QPSK/QAM, $a_{n, I}, a_{n, Q} \in\{-1,+1\}$, while in 16-QAM, $a_{n, I}, a_{n, Q} \in\{ \pm 1, \pm 3\}$, etc.

The bias signal applied to the communicator's transistor gate corresponds to an intermediate frequency (IF) signal and can be represented as

$$
x_{\text {bias }}=\Re\left\{x(t) e^{j 2 \pi f_{\text {sub }} t}\right\}=I(t) \cos \left(2 \pi f_{\text {sub }} t\right)+Q(t) \sin \left(2 \pi f_{\text {sub }} t\right),
$$

where $f_{\text {sub }}$ is the IF (subcarrier) frequency. Assuming that the bias signal level is normalized, i.e. $x_{\text {bias }}(t) \in[-1,+1]$, and the voltage applied to the transistor gate is unipolar, i.e. $V_{\mathrm{b}} \in[0,+1]$ Volts, the relationship between the bias voltage and the IF 
signal is

$$
V_{\mathrm{b}}(t)=\frac{1}{2}+\frac{1}{2} x_{\text {bias }}(t)
$$

Applying a bias voltage to the transistor's gate will modify the voltage-controlled impedance $Z\left(V_{b}(t)\right)$ of the transistor, which in following will modify the reflection coefficient $\Gamma$ at the transistor's source terminal (which is connected to the antenna). The reflection coefficient function over time is

$$
\Gamma\left(V_{b}(t)\right)=\frac{Z\left(V_{b}(t)\right)-Z_{a}^{*}}{Z\left(V_{b}(t)\right)+Z_{a}}
$$

In a simplified scenario, applying a low bias voltage $\left(V_{b}=0\right.$ Volts) to the transistor's gate will turn the transistor 'off', creating an RF-open, which yields a reflection coefficient value $\Gamma(0)=+1$, whereas a high bias voltage $\left(V_{b}=1\right.$ Volt) will create an RF-short which corresponds to a reflection coefficient of $\Gamma(1)=-1$. Furthermore, we will assume a linear relationship between the bias voltage and the reflection coefficient:

$$
\Gamma\left(V_{b}(t)\right)=-2 V_{b}(t)+1=-x_{\text {bias }}(t),
$$

i.e. the IF signal is directly translated to reflection coefficient values. The above assumptions deviate from reality in practical systems because:

- Perfect RF-short and RF-open are difficulty achieved with mmWave transistors, due to parasitics and device insertion loss, which constraint $\Gamma$ to an interval different than $[-1,+1]$. However, with proper signal normalization described in ${ }^{25}$, the reflected signals can be mapped to the $[-1,+1]$ region.

- The relationship between bias voltage and reflection coefficient is not exactly linear, which causes signal distortion. The exact $\Gamma\left(V_{b}\right)$ function has to be characterized with the methods described in $^{25}$, and any non-linearities have to be taken into account with appropriate signal pre-distortion at the communicator, or equalization at the receiver.

Nevertheless, the aforementioned assumptions simplify the analysis of the heterodyne backscatter system. The backscattered signal that is received by a base station/access point and directly down-converted (zero-IF) is:

$$
r(t)=a_{\mathrm{dc}} e^{j \phi_{\mathrm{dc}}}+a_{\bmod } e^{j \phi_{\bmod }} \Gamma\left(V_{b}(t)\right),
$$

where $a_{\mathrm{dc}}, a_{\mathrm{mod}}$ and $\phi_{\mathrm{dc}}, \phi_{\mathrm{mod}}$ are the amplitude scaling and phase rotation terms of the dc and the modulated signal component, respectively. The $r(t)$ signal can be seen as:

$$
r(t)=a_{\mathrm{dc}} e^{j \phi_{\mathrm{dc}}}-a_{\mathrm{mod}} e^{j \phi_{\mathrm{mod}}} x_{\mathrm{bias}}(t)
$$

and is a complex signal that contains the real-valued IF bias signal. To remove the IF subcarrier, a frequency shifting operation followed by a low-pass filter operation is needed. The output signal is

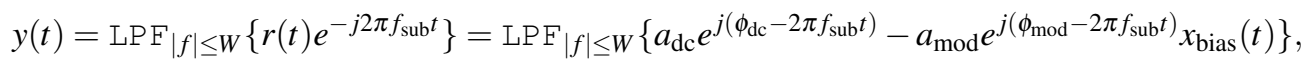

where $\operatorname{LPF}|f| \leq W\{\cdot\}$ denotes an LPF with cutoff frequency $W$, i.e. enough to preserve the frequency content of the band-limited signal of equation (8). Then, the resulting baseband signal is

$$
y(t)=-\frac{1}{2} a_{\bmod } e^{j \phi_{\bmod }}[I(t)+j Q(t)]=-\frac{1}{2} a_{\bmod } e^{j \phi_{\bmod }} x(t),
$$

which allows recovery of the original baseband QAM signal $x(t)$, regardless of the constellation size.

\section{References}

1. Thomas, S. J. \& Reynolds, M. S. A $96 \mathrm{Mbit} / \mathrm{sec}, 15.5 \mathrm{pJ} / \mathrm{bit}$ 16-QAM modulator for UHF backscatter communication. In 2012 IEEE Int. Conf. RFID, 185-190, DOI: https://doi.org/10.1109/RFID.2012.6193049 (2012).

2. Iyer, V., Talla, V., Kellogg, B., Gollakota, S. \& Smith, J. Inter-technology backscatter: Towards internet connectivity for implanted devices. In Proc. of the 2016 ACM SIGCOMM Conf., 356-369, DOI: https://doi.org/10.1145/2934872.2934894 (2016). 
3. Talla, V. et al. Lora backscatter: Enabling the vision of ubiquitous connectivity. Proc. ACM on Interactive, Mobile, Wearable Ubiquitous Technol. 1, 1-24, DOI: https://doi.org/10.1145/3130970 (2017).

4. Belo, D. et al. IQ Impedance Modulator Front-End for Low-Power LoRa Backscattering Devices. IEEE Trans. Microw. Theory Tech. 67, 5307-5314, DOI: https://doi.org/10.1109/TMTT.2019.2941854 (2019).

5. Griffin, J. D. \& Durgin, G. D. Multipath fading measurements for multi-antenna backscatter RFID at $5.8 \mathrm{GHz}$. In 2009 IEEE International Conference on RFID, 322-329, DOI: https://doi.org/10.1109/RFID.2009.4911197 (2009).

6. Bharadia, D., Joshi, K. R., Kotaru, M. \& Katti, S. Backfi: High throughput wifi backscatter. ACM SIGCOMM Comput. Comm. Rev. 45, 283-296, DOI: https://doi.org/10.1145/2829988.2787490 (2015).

7. Tang, A., Kim, Y. \& Chang, M.-C. F. A $65 \mathrm{~nm}$ CMOS $330 \mathrm{Mb} / \mathrm{s}$ microwave backscatter link at 2.4 to $2.9 \mathrm{GHz}$ with ambient blocker cancellation. IEEE Microw. Wirel. Comp. Let. 26, 61-63, DOI: https://doi.org/10.1109/LMWC.2015.2505650 (2015).

8. Pursula, P. et al. Millimeter-wave identification-A new short-range radio system for low-power high data-rate applications. IEEE Trans. Microw. Theory Tech. 56, 2221-2228, DOI: https://doi.org/10.1109/TMTT.2008.2004252 (2008).

9. Yoo, T.-W. \& Chang, K. Theoretical and experimental development of 10 and $35 \mathrm{GHz}$ rectennas. IEEE Trans. Microw. Theory Tech. 40, 1259-1266, DOI: https://doi.org/10.1109/22.141359 (1992).

10. Shinohara, N., Nishikawa, K., Seki, T. \& Hiraga, K. Development of $24 \mathrm{GHz}$ rectennas for fixed wireless access. In IEEE 2011 XXXth URSI Gen. Assem. Sci. Symp., 1-4, DOI: https://doi.org/10.1109/URSIGASS.2011.6050505 (2011).

11. Okba, A., Takacs, A., Aubert, H., Charlot, S. \& Calmon, P.-F. Multiband rectenna for microwave applications. Comptes Rendus Phys. 18, 107-117, DOI: https://doi.org/10.1016/j.crhy.2016.12.002 (2017).

12. Takacs, A., Aubert, H., Fredon, S., Despoisse, L. \& Blondeaux, H. Microwave power harvesting for satellite health monitoring. IEEE Trans. Microw. Theory Tech. 62, 1090-1098, DOI: https://doi.org/10.1109/TMTT.2014.2303425 (2014).

13. Schmid, C. M., Feger, R. \& Stelzer, A. Millimeter-wave phase-modulated backscatter transponder for FMCW radar applications. In 2011 IEEE MTT-S Int.l Microw. Symp., 1-4, DOI: https://doi.org/10.1109/MWSYM.2011.5972773 (2011).

14. Meyer, J., Dao, Q. H. \& Geck, B. Design of a $24 \mathrm{Ghz}$ analog frontend for an optically powered RFID transponder for the integration into metallic components. In 2013 European Microw. Conf., 1531-1534, DOI: https://doi.org/10.23919/EuMC. 2013.6686961 (2013).

15. Kimionis, J., Georgiadis, A. \& Tentzeris, M. M. Millimeter-wave backscatter: A quantum leap for gigabit communication, RF sensing, and wearables. In 2017 IEEE MTT-S Int. Microw. Symp., 812-815, DOI: https://doi.org/10.1109/MWSYM. 2017.8058702 (2017).

16. Matos, D., Daniela da Cruza, M., Jordão, R., Correia \& Carvalho, N. B. Millimeter-wave BiCMOS backscatter modulator for 5G-IoT applications. IEEE Microw. Wirel. Components Lett. 1 - 4, DOI: https://doi.org/10.1109/LMWC.2020.3042709 (2020).

17. Ladan, S., Guntupalli, A. B. \& Wu, K. A high-efficiency $24 \mathrm{GHz}$ rectenna development towards millimeter-wave energy harvesting and wireless power transmission. IEEE Trans. Circuits Syst. I Regul. Pap. 61, 3358-3366, DOI: https://doi.org/10.1109/TCSI.2014.2338616 (2014).

18. Correia, R., Boaventura, A. \& Carvalho, N. B. Quadrature amplitude backscatter modulator for passive wireless sensors in IoT applications. IEEE Trans. Microw. Theory Tech. 65, 1103-1110, DOI: https://doi.org/10.1109/TMTT.2017.2661262 (2017).

19. Correia, R. \& Carvalho, N. B. Ultrafast backscatter modulator with low-power consumption and wireless power transmission capabilities. IEEE Microw. Wirel. Comp. Let. 27, 1152-1154, DOI: https://doi.org/10.1109/LMWC.2017.2760739 (2017).

20. Talla, V., Buettner, M., Wetherall, D. \& Smith, J. R. Hybrid analog-digital backscatter platform for high data rate, battery-free sensing. In 2013 IEEE Topical Conference on Wireless Sensors and Sensor Networks (WiSNet), 1-3 (2013).

21. Daskalakis, S.-N., Assimonis, S. D., Kampianakis, E. \& Bletsas, A. Soil moisture scatter radio networking with low power. IEEE Trans. Microw. Theory Tech. 64, 2338-2346, DOI: https://doi.org/10.1109/TMTT.2016.2572677 (2016).

22. Cook, B. S., Tehrani, B., Cooper, J. R. \& Tentzeris, M. M. Multilayer inkjet printing of millimeter-wave proximity-fed patch arrays on flexible substrates. IEEE Antennas Wirel. Propag. Lett. 12, 1351-1354, DOI: https://doi.org/10.1109/ LAWP.2013.2286003 (2013).

23. Tehrani, B. K., Cook, B. S. \& Tentzeris, M. M. Inkjet-printed 3D interconnects for millimeter-wave system-on-package solutions. In 2016 IEEE MTT-S Int. Microw. Symp., 1-4, DOI: https://doi.org/10.1109/MWSYM.2016.7540084 (2016). 
24. Correia, R. \& Carvalho, N. B. Design of high order modulation backscatter wireless sensor for passive IoT solutions. In 2016 IEEE Wirel. Power Transf. Conf., 1-3, DOI: https://doi.org/10.1109/WPT.2016.7498833 (2016).

25. Kimionis, J. \& Tentzeris, M. M. Pulse shaping: The missing piece of backscatter radio and RFID. IEEE Trans. Microw. Theory Tech. 64, 4774-4788, DOI: https://doi.org/10.1109/TMTT.2016.2623703 (2016).

26. Pozar, D. M. \& Schaubert, D. H. Microstrip Antennas: The Analysis and Design of Microstrip Antennas and Arrays (Wiley-IEEE Press, 1995). ISBN: 978-0-780-31078-0.

27. Pozar, D. Considerations for millimeter wave printed antennas. IEEE Trans. Antennas Propag. 31, 740-747, DOI: https://doi.org/10.1109/TAP.1983.1143124 (1983).

28. Pozar, D. M. Microwave engineering (Fourth Editions, University of Massachusetts at Amherst, John Wiley \& Sons, Inc, 2012).

29. Avago Technologies, Inc. 0.5 to $26 \mathrm{GHz}$ Low Noise E-PHEMT in a Wafer Scale Package (2014). URL: https://docs. broadcom.com/doc/AV02-1082EN.

30. Analog Devices, Inc. MMIC VCO with Half Frequency Output \& Divide-by-16, 23.8- 26.8 GHz (2009). V03.0309, URL: https://www.analog.com/media/en/technical-documentation/data-sheets/hmc739.pdf.

31. Analog Devices, Inc. GaAs pHEMT MMIC 1/2 WATT POWER AMPLIFIER, 24 - 29.5 GHz (2018). V02.0118, URL: https: //www.analog.com/media/en/technical-documentation/data-sheets/hmc863alc4.pdf.

32. RF-Lambda, Inc. Ultra Wide Band Low Noise Amplifier 20 - 40GHz (2018). URL: https://www.rflambda.com/pdf/ lownoiseamplifier/RLNA26G40GB.pdf.

33. Analog Devices, Inc. GaAs, MMIC, I/Q, Downconverter, $20 \mathrm{GHz}$ to $28 \mathrm{GHz}$ (2019). Rev. D, URL: https://www.analog. com/media/en/technical-documentation/data-sheets/HMC977.pdf.

34. Keysight Technologies, Inc. Vector Signal Analysis Basics Application Note (2014). URL: https://www.keysight.com/gb/ en/assets/7018-01220/application-notes/5989-1121.pdf.

35. Shafik, R. A., Rahman, M. S. \& Islam, A. R. On the extended relationships among EVM, BER and SNR as performance metrics. In 2006 Int. Conf. Electr. Comput. Eng., 408-411, DOI: https://doi.org/10.1109/ICECE.2006.355657 (2006).

36. Liu, D. \& Svensson, C. Power consumption estimation in cmos vlsi chips. IEEE J. Solid-State Circuits 29, 663-670, DOI: https://doi.org/10.1109/4.293111 (1994).

37. Proakis, J. G. Digital communications fourth edn, 2001. McGraw-Hill Companies, Inc., New York, NY (1998). URL: http: //www.mhhe.com/engcs/electrical/proakis/.

38. Thomas, S. J., Wheeler, E., Teizer, J. \& Reynolds, M. S. Quadrature amplitude modulated backscatter in passive and semipassive UHF RFID systems. IEEE Trans. Microw. Theory Tech. 60, 1175-1182, DOI: https://doi.org/10.1109/TMTT. 2012.2185810 (2012).

39. Hong, W., Baek, K.-H., Lee, Y., Kim, Y. \& Ko, S.-T. Study and prototyping of practically large-scale mmwave antenna systems for 5g cellular devices. IEEE Comm. Mag. 52, 63-69, DOI: https://doi.org/10.1109/MCOM.2014.6894454 (2014).

40. Occhiuzzi, C., Paggi, C. \& Marrocco, G. Passive rfid strain-sensor based on meander-line antennas. IEEE Transactions on Antennas Propag. 59, 4836-4840, DOI: https://doi.org/10.1109/TAP.2011.2165517 (2011).

41. Niotaki, K., Collado, A., Georgiadis, A., Kim, S. \& Tentzeris, M. M. Solar/electromagnetic energy harvesting and wireless power transmission. Proc.of IEEE 102, 1712-1722, DOI: https://doi.org/10.1109/JPROC.2014.2358646 (2014).

42. Griffin, J. D. \& Durgin, G. D. Complete link budgets for backscatter-radio and rfid systems. IEEE Antennas Propag. Magaz. 51, 11-25, DOI: https://doi.org/10.1109/MAP.2009.5162013 (2009).

43. Kimionis, J., Bletsas, A. \& Sahalos, J. N. Increased range bistatic scatter radio. IEEE Trans. Commun. 62, 1091-1104, DOI: https://doi.org/10.1109/TCOMM.2014.020314.130559 (2014).

\section{Author contributions}

J.K. and A.G. conceived the idea. J.K designed and simulated the backscatter system, performed the measurements, interpreted the results and wrote the initial version of paper. A.G. and M.T. supervised the research and contributed to the general concept and interpretation of the results. S.N.D. preformed simulations, revised and edited the final version of paper. All authors reviewed the manuscript. 


\section{Figures}

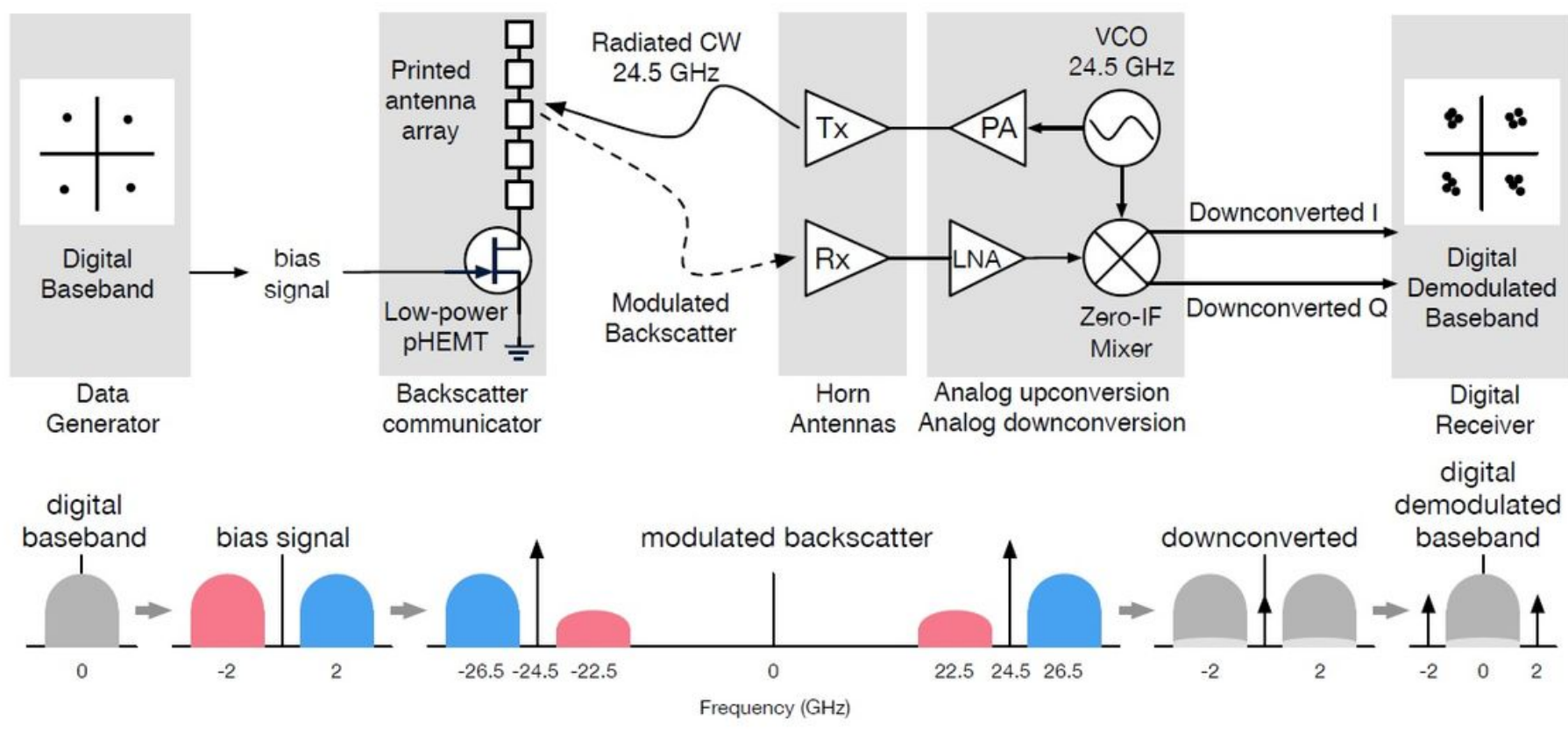

\section{Figure 1}

Top: Gbps-datarate mmWave backscatter system with $24-28 \mathrm{GHz}$ transceiver and single-transistor backscatter communicator. The single-transistor communicator acts as a wireless mixer that is illuminated with an unmodulated continuous wave (CW) signal by the transceiver and mixes high-speed data by controlling the transistor's impedance. The transceiver receives the modulated backscattered signals and demodulates them for digital base-band processing. Bottom: Heterodyne modulation and demodulation of Gbit-datarate mmWave backscatter. Grey lobes correspond to spectra of complex-valued signals.
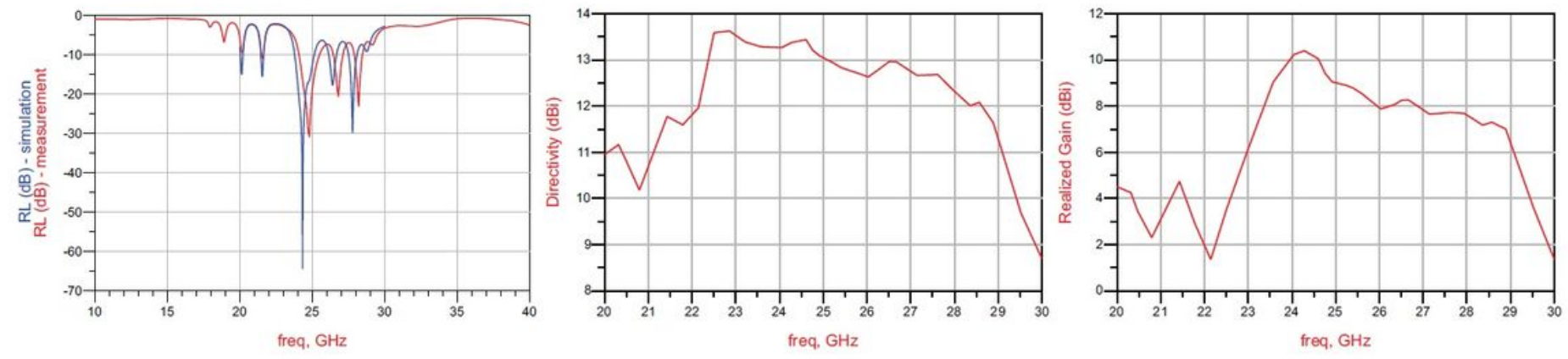

Figure 2

Left: Simulated and measured array return loss $(\mathrm{RL})$. Center: Simulated $5 \times 1$ array directivity. Right: Simulated $5 \times 1$ array realized gain. 

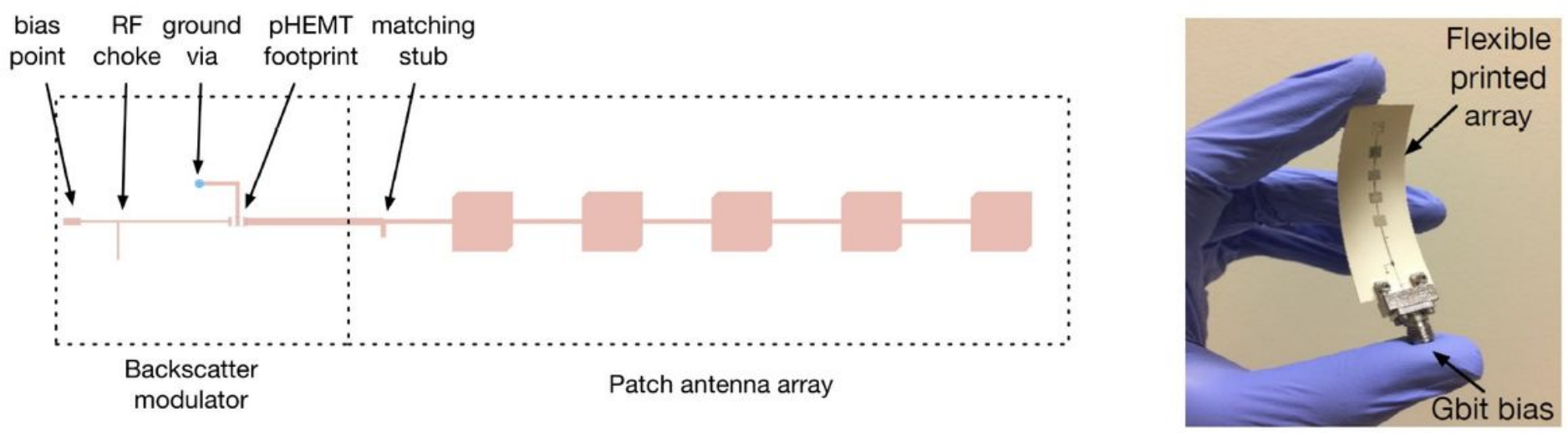

\section{Figure 3}

Left: Physical layout of the backscatter modulator and $5 \times 1$ antenna array physical layout. Right: mmWave backscatter communicator flexible prototype with an integrated pHEMT transistor front-end and patch antenna array.
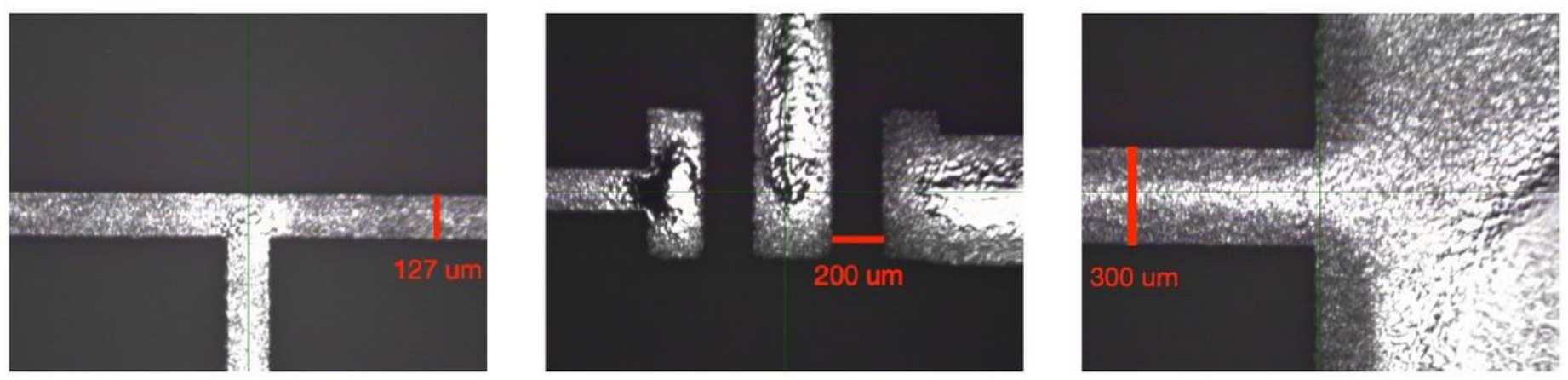

\section{Figure 4}

Microphotographs of inkjet-printed 24.5-GHz patch antenna array and backscatter communication circuit traces. Left: Shunt stub T-junction. Center: 0402 SMD pads for E-pHEMT transistor. Right: RF microstripto-patch transition. 


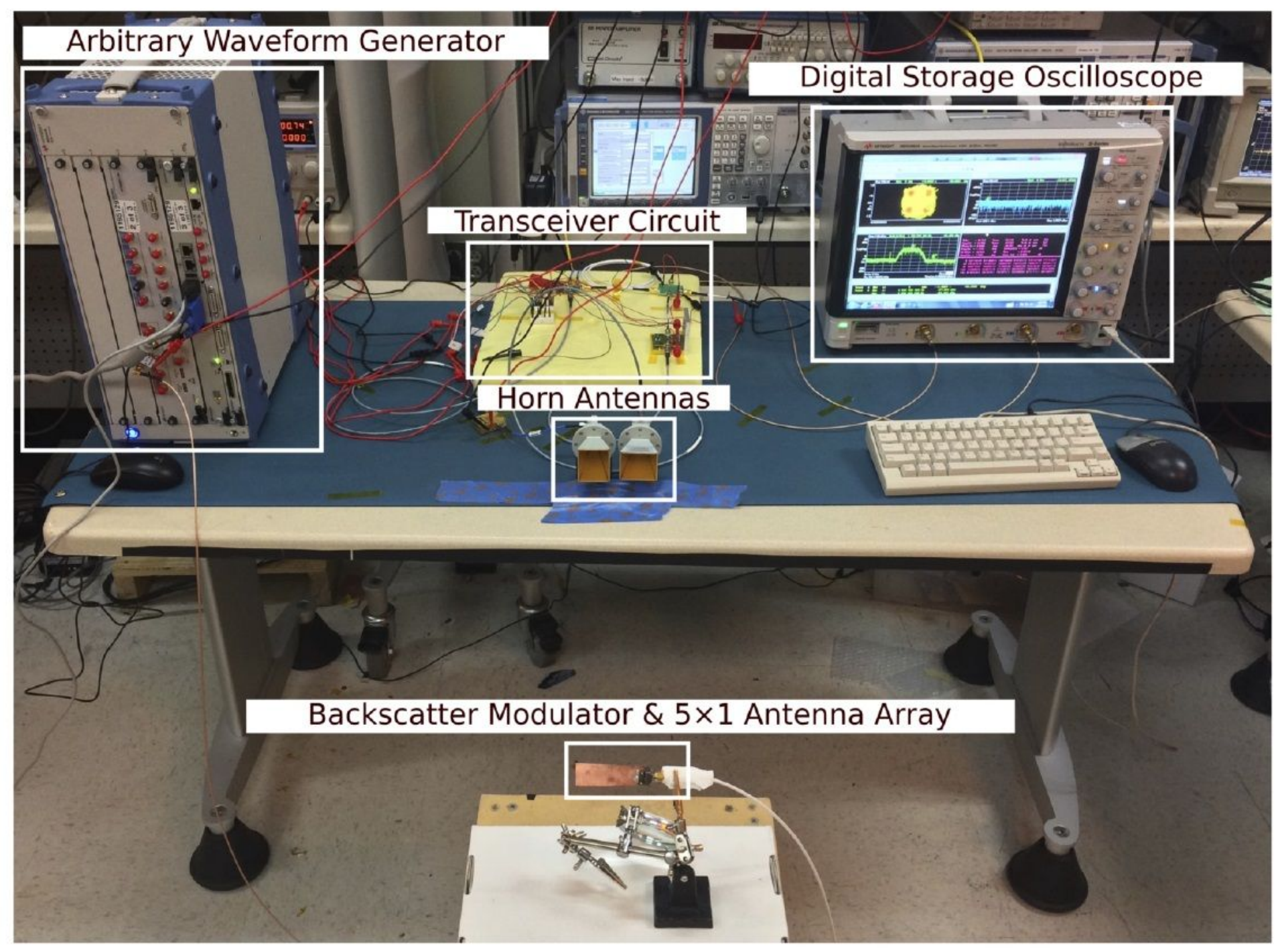

Figure 5

$\mathrm{Gb} / \mathrm{sec} \mathrm{mmWave}$ backscatter setup comprising a waveform generator that drives the backscatter communicator, and a custom transmit and receive chain for illuminating the backscatter communicator, and decoding the reflected modulated signals.
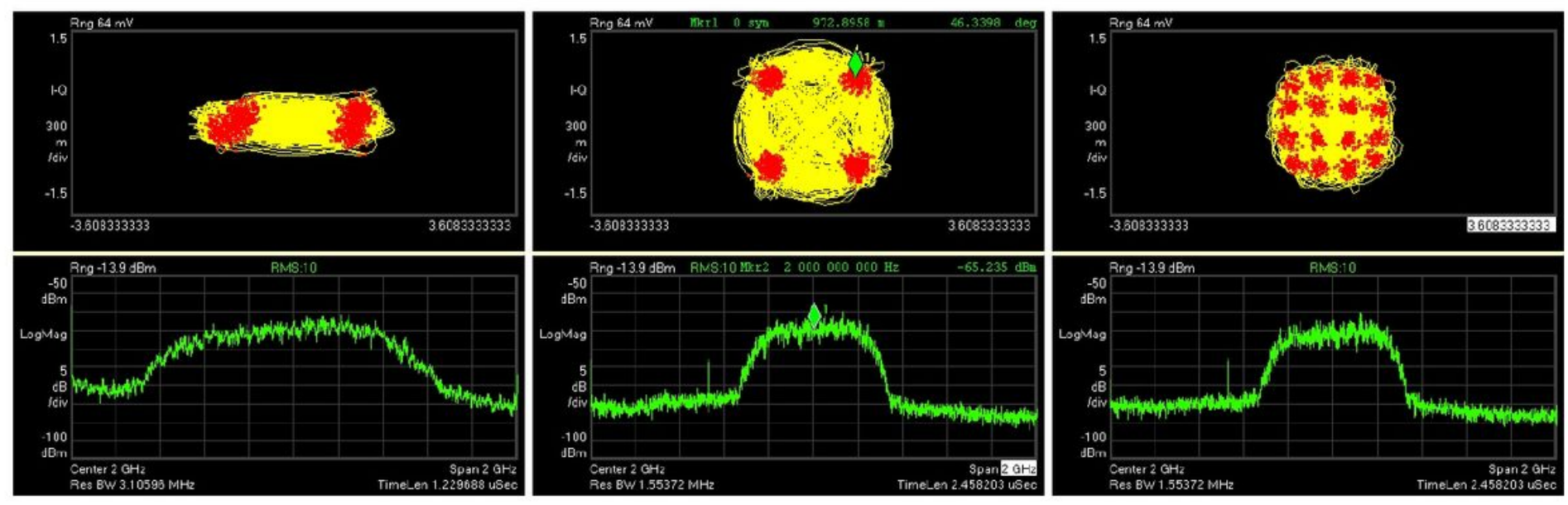
Figure 6

Demodulated Gigabit backscatter constellations and spectra. BPSK, QPSK, and 16-QAM. 